\title{
NICARAGUA: POTENCIAL FARO REGIONAL PARA EL DISEÑO Y EVALUACIÓN DE AGROECOSISTEMAS AGROECOLÓGICOS
}

\section{NICARAGUA: POTENTIAL REGIONAL SPOTLIGHT FOR THE DESIGN AND EVALUATION OF AGROECOLOGICAL AGROECOSYSTEMS}

\section{Salazar-Centeno Dennis}

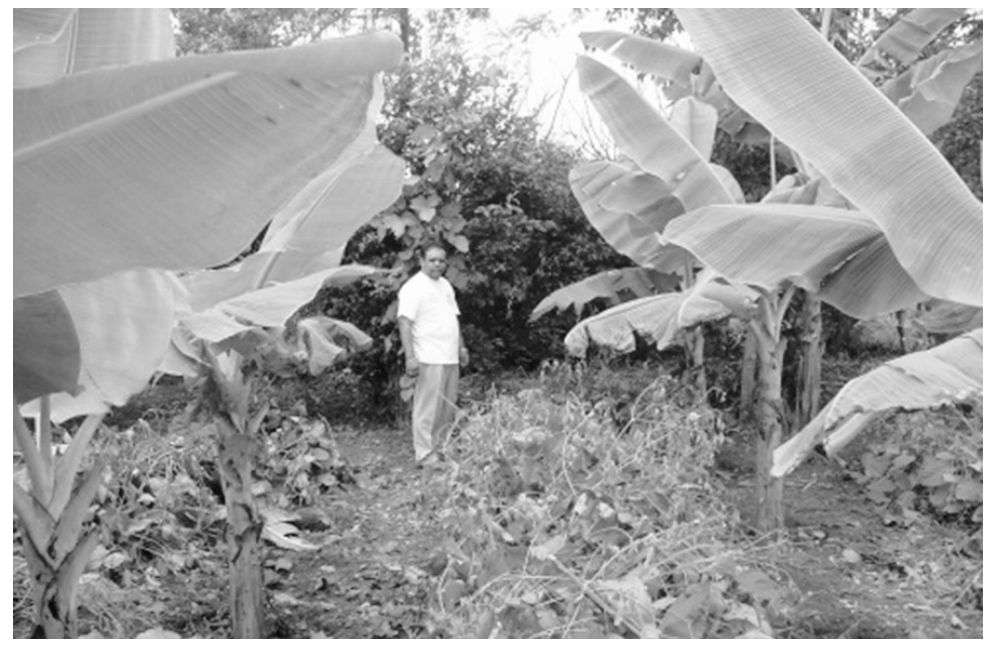

\section{RESUMEN}

En este artículo se expresa la imperiosa necesidad de convertir los sistemas de producción convencionales en fincas agroecológicas como alternativa para que la nueva empresa agraria garantice la implementación de buenas prácticas productivas y de manufactura que contribuyan a ofrecer productos de calidad e inocuos y servicios con un enfoque de calidad total. Desde esta perspectiva, la Universidad Nacional Agraria oferta un programa de formación de talentos humanos a diferentes niveles en agroecología, que consta de cinco grandes proyectos. También, se expresa el andamiaje jurídico nacional actual para implementar la política de la producción agroecológica y orgánica, así como los pasos para el diseño y evaluación de sistemas agroecológicos del presente siglo, cuyos tópicos son: grupos y características de agro ecosistemas, tipos de agro ecosistemas complejos o diversificados, biodiversidad a considerar para el diseño de sistemas diversificados sostenibles en el trópico, ejes a considerar para el diseño de sistemas diversificados sostenibles en el trópico que incluya un manejo integral de los recursos naturales, procesos para el diseño de sistemas diversificados sostenibles en el trópico, pasos y herramientas para el diseño de sistemas diversificados sostenibles en el trópico oferentes de bienes y servicios a la sociedad, ejemplos de sistemas diversificados sostenibles en el trópico oferentes de bienes y servicios, desafíos para el diseño de sistemas diversificados sostenibles en el trópico y la evaluación de sistemas diversificados sostenibles. El propósito es fomentar una agricultura resiliente al cambio climático, productiva y eficiente (energética, económica y biodiversa), pero también que garantice la seguridad alimentaria y nutricional.

\begin{abstract}
This article expresses the urgent need to convert conventional production systems in agro ecological farms as an alternative to the new agrarian enterprise, to ensure the implementation of good manufacturing practices in crop production and contribute to provide safe products and services focused in total quality. From this perspective, the Universidad Nacional Agraria offers a training program for human talents at different levels in agro ecology, which consists of five major projects. Also, the current national legal structure implement agro ecological and organic production policies, to promote ago ecological and organic production in Nicaragua. It is also shows the steps for the design and evaluation of agro-ecological systems, whose topics are: groups and characteristics of agro ecosystems, complex or diversified types of agro ecosystems, biodiversity to be considered for the design of sustainable diversified systems in the tropics, axes to be considered to design sustainable diversified systems in the tropics including integral management of natural resources, processes for designing sustainable diversified systems in the tropics, steps and tools for designing diversified sustainable systems in the tropics which supplied goods and services to society, examples of diversified sustainable systems in the tropics which provided goods and services, challenges for the design of diversified sustainable systems in the tropics and assessment of diversified sustainable systems. The purpose is to foster a resilient agriculture to climate change, productive and efficient (energy, economic and biodivers) as well as that ensure Food security and nutrition
\end{abstract}




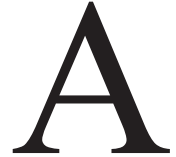

partir del 2006, la Universidad Nacional Agraria (UNA), a través de la Facultad de Agronomía, inició un proceso participativo y de concertación con diferentes actores y promotores del desarrollo del sector agrario para organizar un programa de formación de talentos humanos, a diferentes niveles, con calidad y pertinencia, en AGRO ECOLOGÍA como base para la transformación de los sistemas de producción convencionales en el trópico (Salazar, 2010), de modo que los productos y servicios generados de estas actividades destinados a los mercados extranjeros y nacionales cumplan con una serie de normas establecidas por la Organización Mundial del Comercio (OMC), el Codex Index Alimentarius, por las regulaciones de la Comunidad Económica Europea, Estados Unido, Japón y las legislaciones nacionales. La finalidad es contribuir a la transformación de la agricultura que demanda el presente siglo, respondiendo a un manejo agroecológico de las empresas agrarias de las regiones tropicales (Salazar, 2012; Salazar y Munguía, 2010).

Por otra parte, el planeta azul experimenta un cambio de clima para lo cual debemos prepararnos. Por consiguiente, la agricultura del presente siglo demanda del rediseño de los sistemas convencionales a sistemas agroecológicos de manera que éstos admitan establecer un programa de diversificación productiva, la integración de sus componentes, aprovechar los sinergismos como resultado de la diversificación e integración de sus componentes, ser más eficientes desde el punto de vista energético, económico y de la biodiversidad; y por consiguiente aumentar la capacidad de resiliencia de los agro ecosistemas.

Dicho programa de conversión agroecológica de la nueva empresa agraria debe garantizar la implementación de buenas prácticas productivas y de manufactura que contribuyan a ofrecer productos de calidad e inocuos y servicios con un enfoque de calidad total.

El empresario agrario de la postmodernidad debe combinar e integrar en su sistema alternativas agrícolas, pecuarias, de servicios y de energía renovable. Se trata de una gran diversidad de tecnologías y prácticas adaptadas a las respectivas condiciones agroecológicas, agrarias, sociales y económicas. A mediano plazo, en estos nuevos sistemas de producción será necesario implementar mecanismos que contribuyan a agregar valor a sus productos y que garanticen su trazabilidad. Esta utopía será realidad si en estos países se implementan políticas de estado que abran alianzas a nivel nacional, regional e internacional entre instituciones estatales, no gubernamentales, académicas, organismos donantes, asociaciones de agricultores y los consumidores. Se necesita que los agricultores estén bien informados, manejando procesos ecológicos, de cadena de valor y gerenciales, que les permita incidir en un desarrollo rural integral y sostenible de las zonas rurales del trópico y por consiguiente a mitigar la migración del campo a la ciudad y los cambios climáticos globales. Consecuentemente, las instituciones de educación superior, en el marco de su misión y visión institucional, deben contribuir significativamente a la formación de talentos humanos, generación de conocimientos, alternativas tecnológicas agroecológicas e innovaciones y trabajar hombro a hombro con empresarios agrarios para superar los desafíos arriba descritos.

Proyectos del programa de formación de talentos humanos con calidad y pertinencia. Este programa consta de cinco grandes proyectos (figura 1). Estos son: Dos proyectos que posibiliten realizar investigaciones e innovaciones necesarias para la transformación de la agricultura nicaragüense. Esto se logrará a través de la implementación de una currícula para formar doctores en agro ecología en Nicaragua y en Centro América, que inició en abril del 2013 y cuenta con el apoyo de la Sociedad Científica Latinoamericana de Agroecología (SOCLA); y la implementación de maestrías en: Agro ecología y Desarrollo Sostenible, otra en Sanidad Vegetal y una en Gestión de Medidas Sanitarias y Fitosanitarias. No obstante, se deben aunar esfuerzos para alcanzar la transdisciplinaridad en ambos proyectos de formación académica (doctorado y maestrías).

Un tercer proyecto de actualización dirigido a profesionales y técnicos del sector agrario, que se asienta en la implementación de especialidades y diplomados con enfoque agroecológicos. Los diplomados que se han implementado son: Sistemas de calidad en la producción de café con responsabilidad ambiental, social y empresarial; Cacoacultura Moderna y Calidad, Inocuidad y Trazabilidad de Productos Agrícolas. Adicionalmente, se están formulando un diplomado sobre Desarrollo de Sistemas Sostenibles de Semillas para la Agricultura Familiar y otro sobre Manejo Agroecologíco en Sistemas Agropecuarios.

Un cuarto proyecto es el de mejoramiento continuo de nuestra oferta académica de grado. Este está encaminado a perfeccionar los procesos académicos de esta oferta para los bachilleres que ingresan en nuestra universidad, quienes serán los futuros profesionales para el sector agrario de nuestro país. En este proyecto implementaremos la estrategia de aseguramiento de calidad que está definida en el proyecto educativo de la Universidad que fue aprobado por el Consejo Universitario.

Un último proyecto, pero no menos importante, es de aprendizaje rural, interactuando directamente con técnicos, capataces, administradores, propietarios de las empresas agrarias, promotores agrarios, comunidades rurales, gobiernos y actores locales. Desde esta perspectiva, se han establecido alianzas con instituciones y organización que promueven la producción agroecológica y orgánica en nuestro país, tales como MAONIC (Movimiento de Productoras y Productoras Agroecológicos y Orgánicos de Nicaragua), la UNAG a través del PCaC (Programa Campesino a Campesino), y la 
red de semillas de identidad, etc.

Con este programa de formación de talentos humanos a diferentes niveles, en la Universidad Nacional Agraria, los Docentes, Doctorandos, Maestrantes, profesionales, técnicos, estudiantes, empresarios agrarios y gobiernos locales tendrán la oportunidad de aprender conjuntamente a ser, a saber, a hacer y sobre todo aprender a convivir con sus semejantes y en armonía con la naturaleza, con la pachamama.

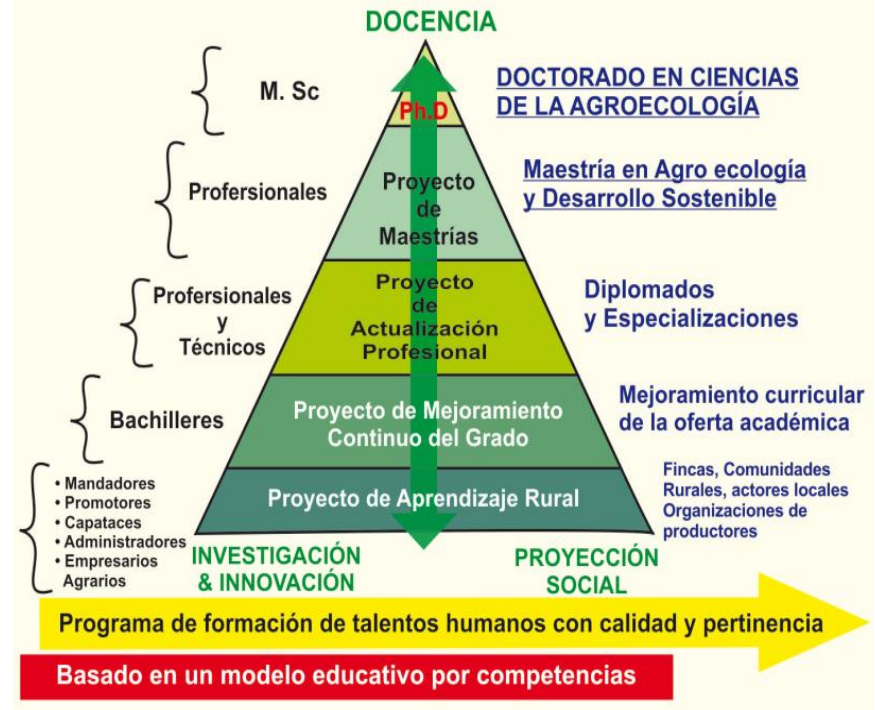

Figura 1: Programa de formación de talentos humanos con calidad y pertinencia.

Andamiaje jurídico y proyecto regional en Nicaragua. El gobierno actual ha desarrollado un andamiaje jurídico para promover la producción agroecológica u orgánica en Nicaragua que fortalece la implementación de este programa de formación de talentos humanos propuesto por la Universidad Nacional Agraria que en alianza con instituciones, organizaciones y redes que promuevan este tipo de producción nos pone como país en una posición propicia para que nuestro terruño sea considerado como un faro agroecológico a nivel regional. Partes de esta plataforma jurídica son:

1. La Ley de Fomento a la Producción Agroecológica u Orgánica (Ley 765) publicada el 5 de julio del 2011 en la GACETA número 124

2. El reglamento de la ley 765 , según el decreto número 022012, aprobado el 23 de enero del 2012 y publicado en La GACETA número 15 del 25 de enero del 2012

3. NTON 11037 - 12 ("Norma Técnica Obligatoria Nicaragüense para la Caracterización, Regulación, y Certificación de Unidades de Producción Agroecológica") y

4. La propuesta de la Política de Fomento y Desarrollo de la Producción Agroecológica
Aunado a la armazón jurídica, el gobierno a través del Ministerio Agropecuario y Forestal (MAGFOR) en el marco del proyecto regional GCP/RLA/195/ BRA, Fortalecimiento de las Políticas Agroambientales en Países de América Latina y el Caribe a través del diálogo e intercambio de experiencias nacionales, financiado por el Ministerio del Medio Ambiente de Brasil y en coordinación con la Organización de las Naciones Unidas para la Alimentación y la Agricultura (FAO) en cinco países de América Latina (Brasil, Chile, Colombia, México y Nicaragua) ha conducido la sistematización de experiencias de aplicación de políticas que fomentan la agricultura ambiental o agroecológica en Nicaragua (MAGFOR 2013).

En síntesis podemos afirmar que este programa de formación de talentos humanos en Agroecología debe contribuir al desarrollo de la agroecología en Nicaragua y en la región como una iniciativa de la UNA en un esfuerzo coordinado y compartido con organizaciones de productores, instituciones académicas (Nacionales, regionales e internacionales), organizaciones no gubernamentales y el gobierno, que articule los diferentes niveles de formación (familias productoras, bachilleres, técnicos, profesionales, maestrantes y doctorandos).

Diseño y evaluación de agro-ecosistemas agroecológicos para el siglo XXI. En el transcurso de formación de talentos humanos en agroecología, a diferentes niveles, se debe explicar el proceso del diseño y evaluación de agro-ecosistemas agroecológicos, cuyos tópicos son:

Grupos y características de agro-ecosistemas. Ramos et $a l$. , (sf) en su artículo sobre "diversidad y tipos de agro ecosistemas: consideraciones para diseño", expresa que si se parte de la idea que un agro sistema es un ecosistema que contiene especies cultivadas agrícolamente, podemos distinguir dos grupos extremos. Uno de éstos, actualmente más extendido, se califica de "moderno", tecnificado o industrial y se caracteriza por requerir subsidio a través de insumos para su mantenimiento y por ser simple estructuralmente (monocultivos, extensivos en el caso de plantaciones). En el otro extremo se encuentran los sistemas tradicionales, típicamente diversificados, que se caracterizan por contener diversidad de especies, que además tienen necesidades pequeñas de insumos externos, debido a su semejanza en estructura y función a los ecosistemas naturales (figura 2). 
Tipos de agro ecosistemas complejos o diversificados. Los tipos de agro ecosistemas complejos o diversificados se muestran en la figura 3, que incluyen sistemas agroforestales, policultivos, cultivos anuales en franjas rotacionales, cultivos de cobertura en plantaciones perennes y también se deben incluir en esta categoría las llamadas fincas integrales, que combinan la producción vegetal, la producción animal, la producción de energía renovable y servicios.

Biodiversidad a considerar para el diseño de sistemas diversificados sostenibles en el trópico. Referente a la biodiversidad debemos entender diferentes definiciones sobre ésta. Las primeras ilustraciones fueron propuestas por Swift y Anderson (1993) de acuerdo a la función que ésta realiza y se ilustran en el la figura 4. Por otra parte, Vandermeer y Perfecto (1995) determinan dos componentes de la biodiversidad en agro ecosistemas, que son la biodiversidad planificada y la asociada (figura 5). Nicholls y Altieri (2002) manifiestan que los componentes de la biodiversidad en agro ecosistemas son tres (biodiversidad productiva, biota funcional y biota destructiva), los que se ilustran en la figura 6. La última clasificación de la biodiversidad según el grado de complejidad del agro ecosistema es propuesta por Vázquez y Matienzo (2006), quienes definieron seis tipos, los que se simplifican en la tabla 1

Ejes a considerar para el diseño de sistemas diversificados sostenibles en el trópico que incluya un manejo integral de los recursos naturales. Los ejes a considerar para el diseño de sistemas diversificados sostenibles en el trópico que incluya un manejo integral de los recursos naturales fueron propuestos por Ramos (1991) y son los siguientes: 1) elevar la calidad de vida de la población local (alimentación, vivienda, vestuario, combustible, salud y recreación), 2) conservar los recursos naturales, 3) desarrollar unidades de producción, integrales y sostenibles, así como establecer y evaluar los sistemas múltiples de producción sostenibles.

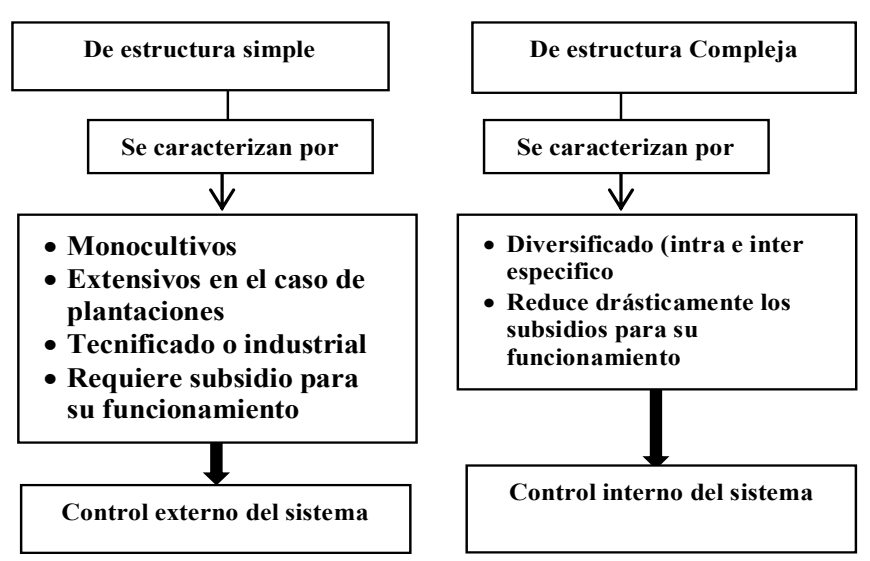

Figura 2. Grupos y características de agro ecosistemas.

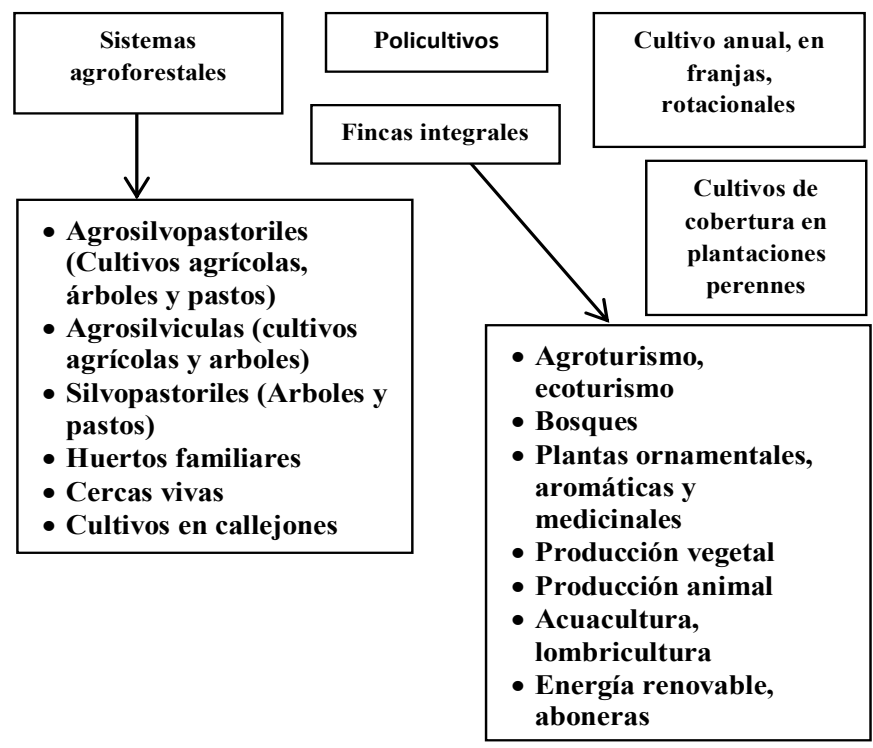

Figura 3: Tipos de agro ecosistemas, complejos y diversificados. 


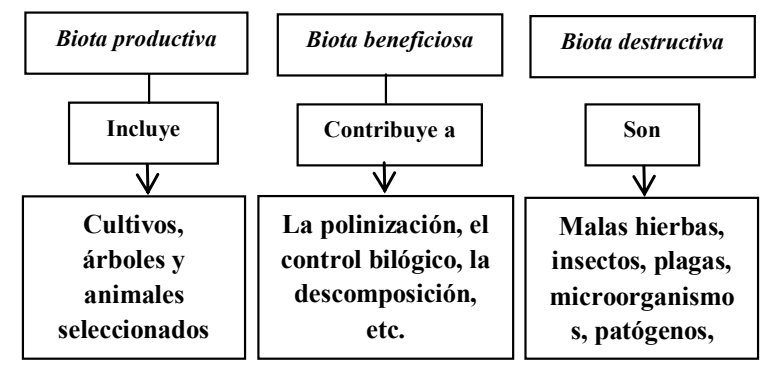

Figura 4. Elementos de la biodiversidad en agro ecosistemas según su función en los cultivos (Swift y Anderson, 1993).
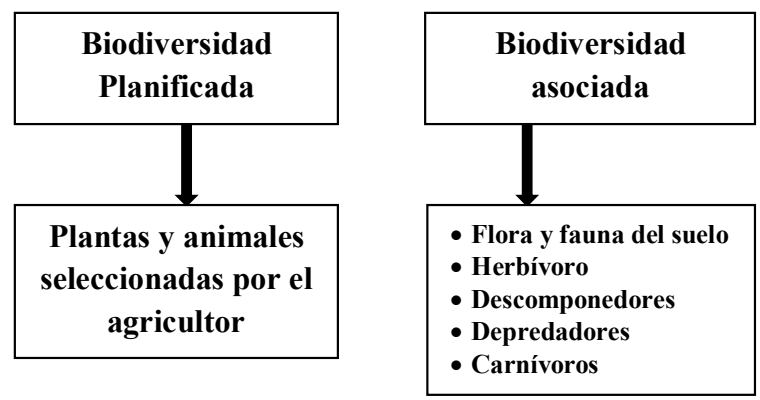

Figura 5. Componentes de la biodiversidad en agro ecosistemas según Vandermeer y Perfecto (1995).

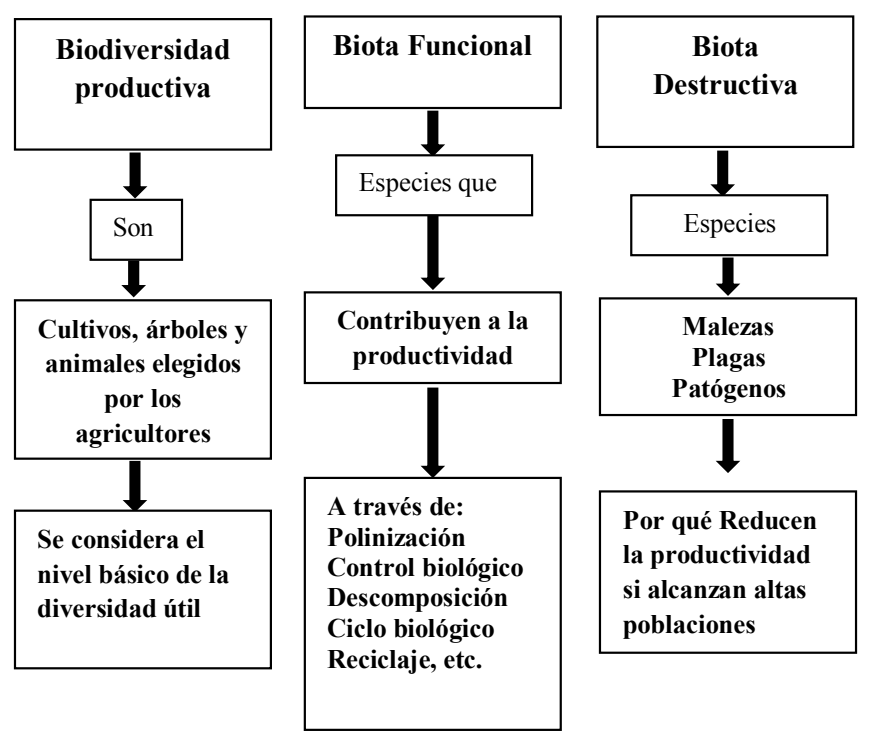

Figura 6. Componentes de la biodiversidad en agro ecosistemas según su función según Nicholls y Altieri (2002).
Tabla 1. Biodiversidad según el grado de complejidad del Agro ecosistema (Vázquez y Matienzo, 2006)

\begin{tabular}{ll}
\hline Tipos & Integrada por \\
\hline Productiva & $\begin{array}{l}\text { Cultivos, árboles, animales } \\
\text { y arreglos de cultivos } \\
\text { Cercas vivas, hierbas } \\
\text { colindantes, arboledas } \\
\text { Puxiliar }\end{array}$ \\
Funcional & $\begin{array}{l}\text { naturales, descomponedores } \\
\text { de la materia orgánica } \\
\text { Micros organismos eficientes, } \\
\text { entomófagos, biopreparados } \\
\text { de micro organismos } \\
\text { Funcional introducida }\end{array}$ \\
& $\begin{array}{l}\text { Plagas que alcanzan } \\
\text { poblaciones altas } \\
\text { Colonizan el agro ecosistema } \\
\text { de ambientes colindantes }\end{array}$ \\
Biota nociva & \\
Asociada &
\end{tabular}

Proceso para el diseño de sistemas diversificados sostenibles en el trópico. Transformar un agro ecosistema de estructura simple a uno de estructura compleja o agroecológico es un proceso del que se puede saber su inicio, pero no su finalización. Este depende de varios factores como: nivel académico y grado de asociatividad del agricultor, capacitación, asistencia técnica, financiamiento, políticas que fomentan este tipo de producción y de las condiciones agroecológicas. Vázquez (2013) establece que dicho proceso para el diseño de sistemas diversificados sostenibles en el trópico consta de : integración, transformación y complejización, las que se resumen en la figura 7.

\begin{tabular}{|c|c|}
\hline \multicolumn{2}{|c|}{$\begin{array}{l}\text { Del manejo del agro ecosistema al diseño y manejo del sistema } \\
\text { de producción }\end{array}$} \\
\hline \multicolumn{2}{|c|}{ Procesos principales } \\
\hline Integración & $\begin{array}{l}\text { - Integración de productos (Sanidad y Nutrición } \\
\text { - Diversificación de rubros productivos } \\
\text { - Integración de rubros productivos (vegetales y } \\
\text { animales }\end{array}$ \\
\hline
\end{tabular}

\begin{tabular}{|l|l|}
\hline Transformación & $\begin{array}{l}\bullet \text { Prelación del suelo (Conservación y mejora } \\
\bullet \text { Diseño espacial de rubros productivos }\end{array}$ \\
\hline
\end{tabular}

\begin{tabular}{|c|l|}
\hline Complejización & $\begin{array}{l}\text { • Complejización de sistemas de cultivo y } \\
\text { crianza } \\
\text { Conexión de la vegetación auxiliar }\end{array}$ \\
\hline
\end{tabular}

Figura 7. Procesos para el diseño de sistemas diversificados sostenibles en el trópico (Vázquez, 2013). 
Pasos y herramientas para el diseño de sistemas diversificados sostenibles en los trópicos oferentes de bienes y servicios a la sociedad. Estas etapas deben planificarse en tiempo y espacio. Martínez et al., (2008) elaboraron una guía de cinco pasos y 12 herramientas para planificar la finca, que se puede utilizar para el diseño de agroecositemas de estructura compleja. En este artículo se propone mantener los cinco pasos pero adicionar una herramienta a como se ilustra en el tabla 2 y considerar los diferentes tipos de biodiversidad propuestas por Vázquez y Matienzo (2006). El propósito de la planificación de fincas diversificadas e integrales es satisfacer las características de los sistemas de producción agroecológicos, que incluyen, además, de la diversificación productiva, la integración de sus componentes, para potenciar los sinergismos de la biodiversidad, y lograr una máxima eficiencia (energética, económica y de la biodiversidad), que contribuya a una mayor resiliencia y por consiguiente mitigar y adaptarnos a los efectos del cambio climático, así como garantizar la seguridad alimentaria y nutricional.

Ejemplos de sistemas diversificados sostenibles en el trópico oferente de bienes y servicios. En el trópico americano, hay ejemplos de estos sistemas agroecológicos sostenibles oferentes de bienes y servicios y Nicaragua no es la excepción. Un ejemplo de este tipo de agro ecosistemas complejos es la finca Los Pinos ubicada en la reserva natural del cerro El Arenal, en la cuenca hidrográfica de Aranjuez, propiedad del Ingeniero Byron Corrales, quién fue fundador de la UNAG y coordinador del $\mathrm{PCaC}$, además es un promotor de la agroecología en diferentes organizaciones. Esta finca es integrante de la cooperativa La Solidaridad y ha sido visitada por estudiantes de grado y posgrado de la Universidad Nacional Agraria para entender su estructura, sus funciones y su manejo.

Tabla 2. Pasos y herramientas para el diseño de sistemas diversificados sostenibles en el trópico considerando los diferentes tipos de biodiversidad (adaptado de Martínez et al., 2008).

\begin{tabular}{|c|c|}
\hline Pasos & Herramientas \\
\hline 1. ¿Quiénes somos y dónde estamos? & 1. Datos de la familia productora y su finca (suelo, topografía, clima) \\
\hline 2. Mapa actual de la finca & 2. Mapa actual de la finca e infraestructura \\
\hline $\begin{array}{l}\text { 3. Registros de los datos climáticos y de } \\
\text { lo que se gasta y lo que se produce } \\
\text { en la finca. }\end{array}$ & $\begin{array}{l}\text { 3. Registros del clima (Temperatura, humedad relativa, precipitación, } \\
\text { viento, etc.) } \\
\text { 4. Registros de los costos de producción por rubro } \\
\text { 5. Formatos para llevar a detalle lo producido } \\
\text { 6. Rendimiento por rubro } \\
\text { 7. Almacenamiento y perdidas pos cosecha. } \\
\text { 8. Consolidado de ingresos agrícolas. } \\
\text { 9. Otros ingresos no agrícolas. } \\
\text { 10. Gastos familiares }\end{array}$ \\
\hline
\end{tabular}

4. Planificar para buscar cómo mejorar la finca.

11. Mapa de la finca AGROECOLÓGICA futura en base a los diferentes tipos de biodiversidad

12. Planificación para el siguiente año

5. Evaluación de lo planificado

13. Evaluación del plan de la finca AGROECOLÓGICA del año próximo
Los postulados del sistema de producción de la finca Los Pinos son los siguientes:

a. El café no funciona como un sistema aislado de nuestra sociedad.

b. Buscamos una integración entre la producción y la protección del medio ambiente.

c. Nuestras prácticas son biodinámicas y aprovechamos toda la energía que nos rodea.

d. Todos nuestros cultivos y animales están interconectados, produciendo la energía necesaria para el consumo de la familia y para la vida del suelo.

e. Nuestra producción se basa en el desarrollo de nuestras comunidades y en la protección de nuestras riquezas naturales.

f. El mejoramiento de la calidad, es un manejo integral de todo el proceso de la producción, hasta el envío del café.

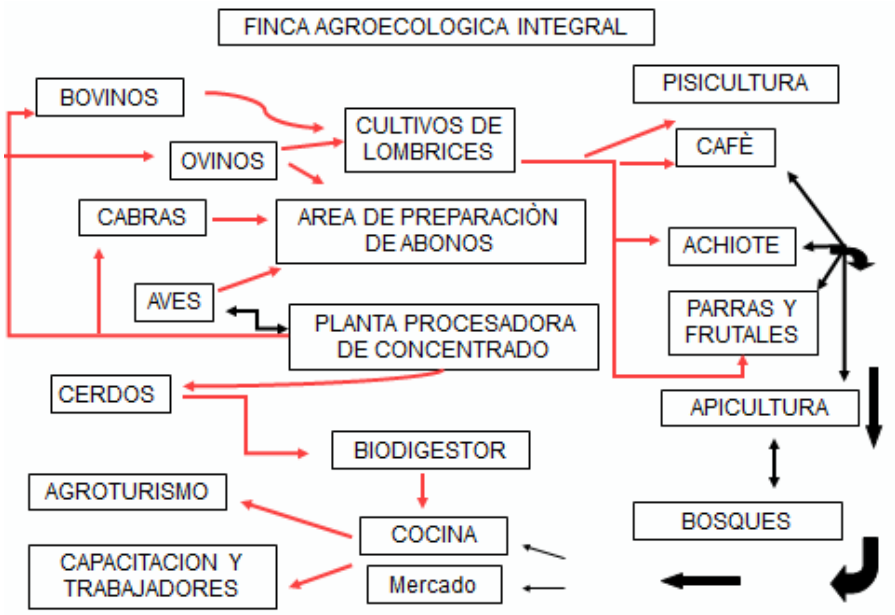

Figura 8: Esquema realizado por estudiantes de un curso de agroecología al visitar la finca La Canavalia en el año 2007.

Otro ejemplo nicaragüense es la finca La Canavalia, cuyo responsable, el Dr. Cesar Tercero, quien nos explicó, en una visita que realizamos hace varios años, los componentes y la integración de éstos en esta unidad de producción agroecológica. Este ejemplo nos muestra que las excretas de los animales tienen usos específicos y combinan la producción animal, vegetal y servicios (figura 8). Esta finca está ubicada en el municipio de San Ramón, Matagalpa, y pertenece a la Asociación para la Diversificación y el Desarrollo Agrícola Comunal (ADDAC).

Basados en estas dos experiencias de nuestro país, podemos deducir que para la gerencia de sistemas agroecológicos con 
enfoque empresarial es fundamental contar con talento humano calificado, tener relaciones con organizaciones gremiales y financieras, así como constar con instancias técnicas de carácter privadas y/o públicas.

Desafíos para el diseño de sistemas diversificados sostenibles en el trópico. Para fomentar masivamente el diseño de sistemas diversificados sostenibles en el trópico es fundamental superar varios desafíos que consisten en:

a. Implementar en estos sistemas prácticas administrativas b. Fomentar una agricultura resiliente al cambio climático, productiva y eficiente (energética, económica y biodiversidad)

c. Ofertar productos y servicios con calidad, inocuos, trazables; y con agregación de valor

d. Planificar participativamente para la transformación hacia sistemas agroecológicos

e. Disponer de un plan de negocio o inversión

f. Tener una visión empresarial

g. Fomentar la asociatividad de las familias productoras

h. Disponer de mercados nacionales e internacionales

i. Certificación participativa, es decir crear mecanismos

alternativos de certificación para los mercados locales.

Evaluación de sistemas diversificados sostenibles en los trópicos oferentes de bienes y servicios. Una vez que el agricultor tomó la decisión de transformar su sistema de producción a un sistema agroecológico debe constatar el grado de evolución de éste a través del tiempo o comparar su progreso con otro sistema agroecológico en el mismo tiempo.

La herramienta metodológica que se usa en estos casos es El Marco para la Evaluación de Sistemas de Manejo de recursos naturales incorporando Indicadores de Sustentabilidad (MESMIS). Es un instrumento metodológico desarrollado por Masera et al., (1999) que:

a. Ayuda a evaluar la sustentabilidad de sistemas de manejo de recursos naturales, con énfasis en el contexto de los agricultores y en el ámbito local, desde la parcela hasta la comunidad.

b. Brinda una reflexión crítica destinada a mejorar las posibilidades de éxito de las propuestas de sistemas de manejo alternativos y de los propios proyectos involucrados en la evaluación.

c. Busca entender de manera integral las limitantes y posibilidades para la sustentabilidad de los sistemas de manejo que surgen de la intersección de procesos ambientales con el ámbito social y económico.

d. Permite comparar a los sistemas de manejo en términos de su sustentabilidad, ya sea mediante la confrontación de uno o más sistemas alternativos con un sistema de referencia (comparación transversal) o bien mediante la observación de los cambios de las propiedades de un sistema de manejo particular a lo largo del tiempo (comparación longitudinal).

e. Presenta una estructura flexible para adaptarse a diferentes niveles de información y capacidades técnicas disponibles localmente. Asimismo, propone un proceso de evaluación participativo que enfatiza dinámicas de grupo y una retroalimentación continua del equipo evaluador.

f. Constituye una herramienta en desarrollo. La experiencia de su aplicación permitirá mejorar el propio marco. En este sentido, debe entenderse al MESMIS como un método para organizar (mas no agotar) la discusión sobre sustentabilidad y la forma de hacer operativo el concepto.

En Nicaragua esta metodología ha sido aplicada en proyectos de desarrollo y en la formación de talentos humanos a nivel de grado y posgrado. Un ejemplo de la aplicación de esta metodología en nuestro país es el estudio realizado por Arnés (2011), quien evaluó la sostenibilidad de los campesinos de montaña en San José de Cusmapa.

\section{CONCLUSIÓN}

Nicaragua tiene el potencial para ser un faro a nivel regional para el diseño y evaluación de agro ecosistemas agroecológicos, dado que cuenta con un programa de formación de talentos humanos en agroecología como base para la transformación de los sistemas de producción convencionales en el trópico. Talentos humanos a nivel nacional, un andamiaje jurídico, voluntad política del gobierno para promover la producción agroecológica y orgánica, organizaciones, instituciones y agricultores que cuentan con experiencia desde la década de los ochenta para hacer realidad este desafío, como son los promotores del pcac, la Red de Semillas de Identidad y más recientemente MAONIC, etc. El reto es articular estos vigores dispersos para gestionar los recursos y los conocimientos necesarios y contribuir a la transformación del sector agrario nacional y regional a través de la implementación de principios y estrategias para el diseño de sistemas agrarios sostenibles que demanda la agricultura del siglo XXI. Desde esta óptica, Altieri (2001) plantea que la Agroecología provee una guía para desarrollar agro ecosistemas que tomen ventajas de los efectos de la integración de la biodiversidad de plantas y animales. Tal integración aumenta las complejas interacciones y sinergismos y optimiza las funciones y procesos del agro ecosistema tales como la regulación biótica de organismos perjudiciales, reciclado de nutrientes y la producción y acumulación de biomasa, permitiendo así al agro ecosistema autoregular su propio funcionamiento. Por consiguiente, consiste en fomentar una agricultura resiliente al cambio climático, productiva y eficiente (energética, económica y biodiversa), así como garantizar la seguridad alimentaria y nutricional. 


\section{REFERENCIAS BIBLIOGRÁFICAS}

Altieri, M. 2001. Agroecología: principios y estrategias para diseñar sistemas agrarios sustentables.

Arnés, E. 2011. Desarrollo de la metodología de evaluación de sostenibilidad de los campesinos de montaña en San José de Cusmapa, Nicaragua. Master de Tecnología Agroambiental para una Agricultura Sostenible. Universidad Politécnica de Madrid, 48 p.

MAGFOR. 2013. Ministerio Agropecuario y Forestal. Identificación, sistematización y análisis de experiencias de aplicación de políticas que fomentan la agricultura ambiental en Nicaragua: fincas agroecológicas de productores y productoras que contribuyen a la soberanía alimentaria y nutricional y a la erradicación de la pobreza en Nicaragua/norma técnica de producción agroecológica u orgánica (NTON 11/03/12), Managua, Nicaragua. 27 p.

Martínez, CJ; Franklin Martínez, F; Matamoros, E; Rocha, A. 2008. 5 pasos y 12 herramientas para planificar la finca. Managua: EDISA, $70 \mathrm{p}$.

Masera, O; Astier, M.; López-Ridaura, S. 1999. "Sustentabilidad y Manejo de Recursos Naturales. El marco de Evaluación MESMIS” Ed. Mundiprensa - GIRA - UNAM. México.

Nicholls, C; Altieri, M. 2002. Biodiversidad y diseño agroecológico: un estudio de caso de manejo de plagas en viñedos. Manejo Integrado de Plagas y Agroecología (Costa Rica) No. 65. p. 50 - 64

Ramos, J M; Rodríguez, S; Arévalo, J (sf). Diversidad y tipos de agroecosistemas: Consideraciones para diseño. www.valsalice.edu co/.../Diversidad\%20y\%20tipos\%20de\%20agroecosist.

Ramos, J M. 1991. Modelo de manejo integral de un ejido con clima cálido-húmedo. Proyecto de Investigación, UAM-Xochimilco, México.

Salazar, D. 2012. Estrategia para implementar un programa de formación de talentos humanos con calidad y pertinencia en el sector agroalimentario y recursos naturales. En: Memoria del Octavo Congreso Internacional de Educación Superior. Universidad 2012 , Cuba; II Taller Internacional "Universidad, Seguridad y Soberanía Alimentaria. ALI 018.

Salazar, D; Munguía, R. 2010. Estrategia para la elaboración de la curricula del diplomado en sistemas de calidad en la producción de café con responsabilidad ambiental, social y empresarial. En: Alemán et al, 2011. Innovaciones en las universidades nicaragüenses. P: $163-171$.

Salazar, D. 2010. La formación agroecológica: base para la transformación de los sistemas de producción convencionales en el trópico. En: memoria del XVII congreso científico internacional del Instituto Nacional de Ciencias Agrícola de Cuba.

Swift, MJ; Anderson, JM. 1993. Biodiversity and ecosystem function in agroecosystems. In: Schultze, E., Mooney, H.A. (Eds.), Biodiversity and Ecosystem Function. Spinger, NewYork, p. 57-83.

Vandermeer, J; Perfecto, I. 1995. Breakfast of biodiversity: The truth about rain forest destruction. Oaklan, California. The Institute of Food and Development Policy.

Vásquez, L. 2013. Diseño y manejo agroecológico del sistema de producción: enfoque holístico para suprimir poblaciones de organismos nocivos. Conferencia en el doctorado en agroecología, Universidad Nacional Agraria, Managua, Nicaragua.

Vázquez, L; Matienzo, Y. 2006. Metodología para la caracterización rápida de la diversidad biológica en las fincas, como base para el manejo agroecológico de plagas. Instituto de Investigaciones de Sanidad Vegetal (INISAV), Ministerio de la Agricultura, Cuba. 